
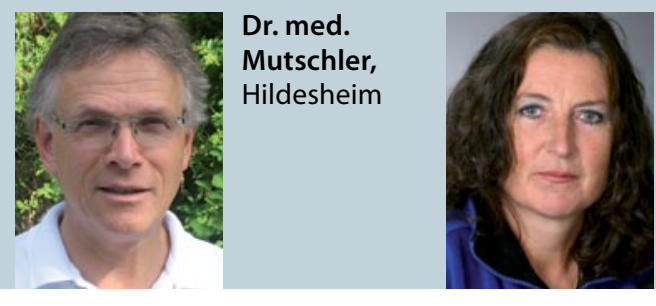

Dr. med.

Kirsten

Stollhoff,

Hamburg

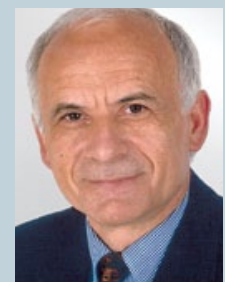

Prof. Dr. med.

Alfred Wirth,

Bad Rothen-

felde

\title{
Finnische Fälle von Narkolepsie nach H1N1-Impfung
}

\begin{abstract}
Vor einem Jahr hat das finnische Gesundheitsinstitut die Zwischenergebnisse einer Kohortenstudie veröffentlicht, nach denen die H1N1-Impfung mit Pandemrix ${ }^{\circledR}$ mit einem neunfach erhöhten Risiko für Narkolepsie assoziiert ist. Jetzt liegt die Endauswertung durch Wissenschaftler aus Helsinki vor.
\end{abstract}

$\mathrm{K}^{\mathrm{u}}$

urz nach Beginn der Schweinegrippepandemie und der Impfung gegen das Virus in Finnland im Jahr 2010 wurde ein plötzlicher Anstieg der Zahl der Patienten mit Narkolepsie registriert. Es war nur die Altersgruppe der 4- bis 19-Jährigen betroffen. Für die Impfung wurde ausschließlich Pandemrix ${ }^{\circledast}$ verwendet. Der Impfstoff gegen Influenza A/H1N1 enthält das Adjuvans AS03, eine Wasser-in-Öl-Emulsion mit Squalen, Vitamin E und Polysorbat 80.

In einer retrospektiven Kohortenstudie wurden die Daten aller 4- bis 19-Jährigen analysiert. Das Follow-up wurde zeitlich begrenzt: Es begann am 1. Januar 2009 und endete am 15. August 2010, also genau einen Tag bevor in der finnischen Presse über das Thema Narkolepsie nach der Schweinegrippeimpfung diskutiert wurde. Anlass der Medienberichte waren damals Kinder mit Narkolepsie in Schweden. Die Erkrankungen standen in zeitlichem Zusammenhang mit der Pandemrix ${ }^{\circledast}$-Impfung. Die Wissenschaftler schlossen so aus, dass die Medienberichte die Angaben zur Narkolepsierate nach der Impfung verzerrten.

Insgesamt 71 Narkolepsieerkrankungen wurden in der Gruppe der 4- bis 19-Jährigen 2009/2010 erstmals diagnostiziert, und zwar anhand des Schlüssels ICD10-G47.4. Insgesamt 67 Erkrankungen wurden bestätigt. Die statistische
Auswertung ergab schließlich eine Narkolepsieinzidenz von 9,0/100.000 Personenjahren in der Gruppe der Geimpften und von 0,7/100.000 Personenjahren in der Gruppe der Ungeimpften. Das entspricht einem relativen Risiko von 12,7. Nach Angaben der finnischen Wissenschaftler bestätigen die Ergebnisse dieser Studie Daten aus Schweden, nach denen die Impfung mit Pandemrix ${ }^{\circledast}$ mit einem um das Siebenfache erhöhten Narkolepsierisiko assoziiert ist.

Eine Erklärung für die Assoziation zwischen Schweinegrippeimpfung und Narkolepsie könnte nach Angaben der Wissenschaftler der immunmodulierende Effekt der Impfung sein sowie die Tatsache, dass Narkolepsie stark mit dem HLA-Allel DQB1 ${ }^{\star} 0602$ assoziiert ist. Das Allel kommt doppelt so häufig in nord- wie in südeuropäischen Ländern vor. Die finnischen Forscher vermuten, dass die Impfung mit der adjuvantierten Vakzine eher einen bereits begonnenen Krankheitsprozess verstärkt als eine Narkolepsie in Zusammenhang mit einer Autoimmunerkrankung auslöst. Von Squalen, das in Impfstoffen enthalten ist, sei aus Tiermodellen bekannt, dass es die Entstehung einer Autoimmunkrankheit fördern kann. Epidemiologische Daten bestätigten diesen Zusammenhang bei Menschen allerdings nicht.

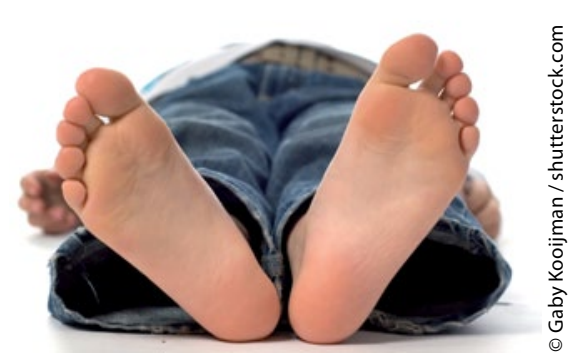

In Finnland kam es nach der Impfung mit der H1N1-Vakzine gehäuft zu Fällen von Narkolepsie.

Dringend zu prüfen ist nach Angaben der Wissenschaftler jetzt, ob die Assoziation zwischen der Impfung gegen Schweinegrippe und Narkolepsie auch in anderen Bevölkerungsgruppen nachweisbar ist.

In Deutschland, wo auch einige wenige Narkolepsieverdachtsfälle nach der Impfung mit Pandemrix ${ }^{\circledast}$ gemeldet wurden, läuft inzwischen eine epidemiologische Studie zur Assoziation zwischen der Impfung gegen H1N1 und dem Auftreten von Narkolepsie. An der Studie sind das Paul-Ehrlich-Institut und die Deutsche Gesellschaft für Schlafforschung und Schlafmedizin (DGSM) beteiligt. Der Vorstand der DGSM bittet alle schlafmedizinischen Zentren zur Mitarbeit.

Nohynek $\mathrm{H}$ et al. AS03 adjuvanted AH1N1 vaccine associated with an abrupt increase in the incidence of childhood narcolepsy in Finland. PLoS ONE 2012; 7: e33536

Kommentar: Diese Studie bestätigt wieder einmal die Erkenntnis, dass nach Neuzulassungen von Medikamenten erst im "realen Leben" alle möglichen Nebeneffekte und Probleme erfasst werden (können). Um so wichtiger ist die genaue (Nach-)Beobachtung dieser Präparate und ein gut ausgebautes Berichtsystem zur raschen Information aller Beteiligten.

Dr. Ulrich Mutschler/ Peter Leiner 